\title{
DOT Tomography of the Solar Atmosphere VII. Chromospheric Response to Acoustic Events
}

\author{
R.J. Rutten · B. van Veelen • P. Sütterlin
}

Received: 8 October 2007 / Accepted: 3 January 2008 / Published online: 19 February 2008

(C) The Author(s) 2008. This article is published with open access at Springerlink.com

\begin{abstract}
We use synchronous movies from the Dutch Open Telescope sampling the $\mathrm{G}$ band, $\mathrm{Ca} I \mathrm{H}$, and $\mathrm{H} \alpha$ with five-wavelength profile sampling to study the response of the chromosphere to acoustic events in the underlying photosphere. We first compare the visibility of the chromosphere in $\mathrm{Ca}$ II $\mathrm{H}$ and $\mathrm{H} \alpha$, demonstrate that studying the chromosphere requires $\mathrm{H} \alpha$ data, and summarize recent developments in understanding why this is so. We construct divergence and vorticity maps of the photospheric flow field from the G-band images and locate specific events through the appearance of bright $\mathrm{Ca}$ II $\mathrm{H}$ grains. The reaction of the $\mathrm{H} \alpha$ chromosphere is diagnosed in terms of brightness and Doppler shift. We show and discuss three particular cases in detail: a regular acoustic grain marking shock excitation by granular dynamics, a persistent flasher, which probably marks magnetic-field concentration, and an exploding granule. All three appear to buffet overlying fibrils, most clearly in Dopplergrams. Although our diagnostic displays to dissect these phenomena are unprecedentedly comprehensive, adding even more information (photospheric Doppler tomography and magnetograms along with chromospheric imaging and Doppler mapping in the ultraviolet) is warranted.
\end{abstract}

Keywords Chromosphere · Granulation · Oscillations · Waves

Helioseismology, Asteroseismology and MHD Connections Guest Editors: Laurent Gizon and Paul Cally.

Electronic supplementary material The online version of this article (http://dx.doi.org/10.1007/s11207-008-9116-9) contains supplementary material, which is available to authorized users.

R.J. Rutten $(\varangle) \cdot$ B. van Veelen · P. Sütterlin

Sterrekundig Instituut, Utrecht University, Utrecht, The Netherlands

e-mail: r.j.rutten@astro.uu.nl

B. van Veelen

e-mail: b.vanveelen@astro.uu.nl

P. Sütterlin

e-mail: p.suetterlin@astro.uu.nl

R.J. Rutten

Institute of Theoretical Astrophysics, University of Oslo, Oslo, Norway 


\section{Introduction}

"Acoustic events" (or "seismic events") is the term used by P. Goode and co-workers (Stebbins and Goode, 1987; Goode, Gough, and Kosovichev, 1992; Restaino, Stebbins, and Goode, 1993; Rimmele et al., 1995; Goode et al., 1998; Strous, Goode, and Rimmele, 2000) to describe localized, small-scale happenings in the granulation that produce excessive amounts of upward-propagating acoustic waves in the upper photosphere, with the claim that these indicate the kinetic sources of the global $p$ modes, "the smoke from the fire exciting the solar oscillations" (Goode, 1995). Their technique was to sample the Doppler modulation of a suitable spectral line at different profile heights through Fabry - Perot imaging with rapid wavelength shifting, and then isolate the surface locations with the largest oscillatory amplitude at five-minute periodicity and upward-propagating phase. Their result was that this measure of "acoustic flux" tends to be maximum above intergranular lanes, in particular those in which a small granule has vanished in so-called granular collapse.

Acoustic excitation through granular dynamics was also addressed theoretically and through numerical hydrodynamics simulations by Rast (1995, 1999), Skartlien and Rast (2000), and Skartlien, Stein, and Nordlund (2000), confirming the picture of small vanishing granules, especially at mesogranular downdraft boundaries, acting as "collapsars" to excite upward-propagating waves, in particular at three-minute periodicity corresponding to the photospheric acoustic cutoff frequency.

In this paper we address the reaction of the overlying chromosphere to such events.

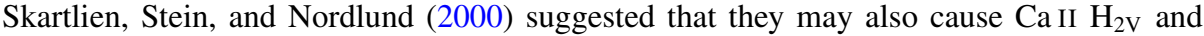
$\mathrm{K}_{2 \mathrm{~V}}$ cell grains, an enigma for many decades (see the review by Rutten and Uitenbroek, 1991) that was eventually solved by Carlsson and Stein (1994, 1997), who identified them as marking upward-propagating acoustic shocks. The announcement by Goode (2002) that indeed acoustic events cause such acoustic grains in the chromosphere was followed up by Hoekzema, Rimmele, and Rutten (2002), who found that, although extreme acoustic events do tend to correlate with the subsequent appearance of exceptionally bright $\mathrm{Ca}$ II $\mathrm{K}_{2 \mathrm{~V}}$ internetwork grains, this correspondence is far from a one-to-one correlation.

We now turn to $\mathrm{H} \alpha$ rather than $\mathrm{H}$ and $\mathrm{K}$ as diagnostic of the chromospheric response to acoustic events. It is important to note and to explain that $\mathrm{H} \alpha$ is a much better chromospheric diagnostic than the $\mathrm{Ca}$ II $\mathrm{H}$ and $\mathrm{K}$ lines are. This is not the case for simple Saha-Boltzmann LTE partitioning in which $\mathrm{H}$ and $\mathrm{K}$ have larger opacity than $\mathrm{H} \alpha$ throughout the atmosphere (see Figure 6 of Leenaarts et al., 2006, and the second student exercise at http://www.astro. uu.nl/ rutten/education/rjr-material/ssa), and it is also not the case in the standard NLTE statistical and hydrostatic equilibrium VAL modeling of Vernazza, Avrett, and Loeser (1973, 1976, 1981) in which the line center of Ca II K is formed higher than the line center of $\mathrm{H} \alpha$ (see the celebrated Figure 1 of Vernazza, Avrett, and Loeser, 1981). We use displays from older data here to argue that the real Sun does not conform.

Figure 1 compares Dutch Open Telescope (DOT) images using the two diagnostics. The upper image is taken in $\mathrm{Ca}$ II $\mathrm{H}$ and shows a mixture of mid- and upper photospheric contributions (reversed granulation and acoustic grains) and chromospheric contributions (bright plage, network, and straws in hedge rows as described by Rutten, 2007). The lower panel shows the same area observed in $\mathrm{H} \alpha$ line center. Comparison of the two demonstrates unequivocally that in such filter imaging $\mathrm{H} \alpha$ presents a much more complete picture of the chromosphere. In our opinion, the chromosphere is best defined as the collective of ubiquitous fibrils seen in this line. Most of these are internetwork-spanning structures, which probably outline magnetic canopies. Only truly quiet internetwork areas (some of which are seen in the lower part of the image) are not masked by such canopy fibrils but show much 

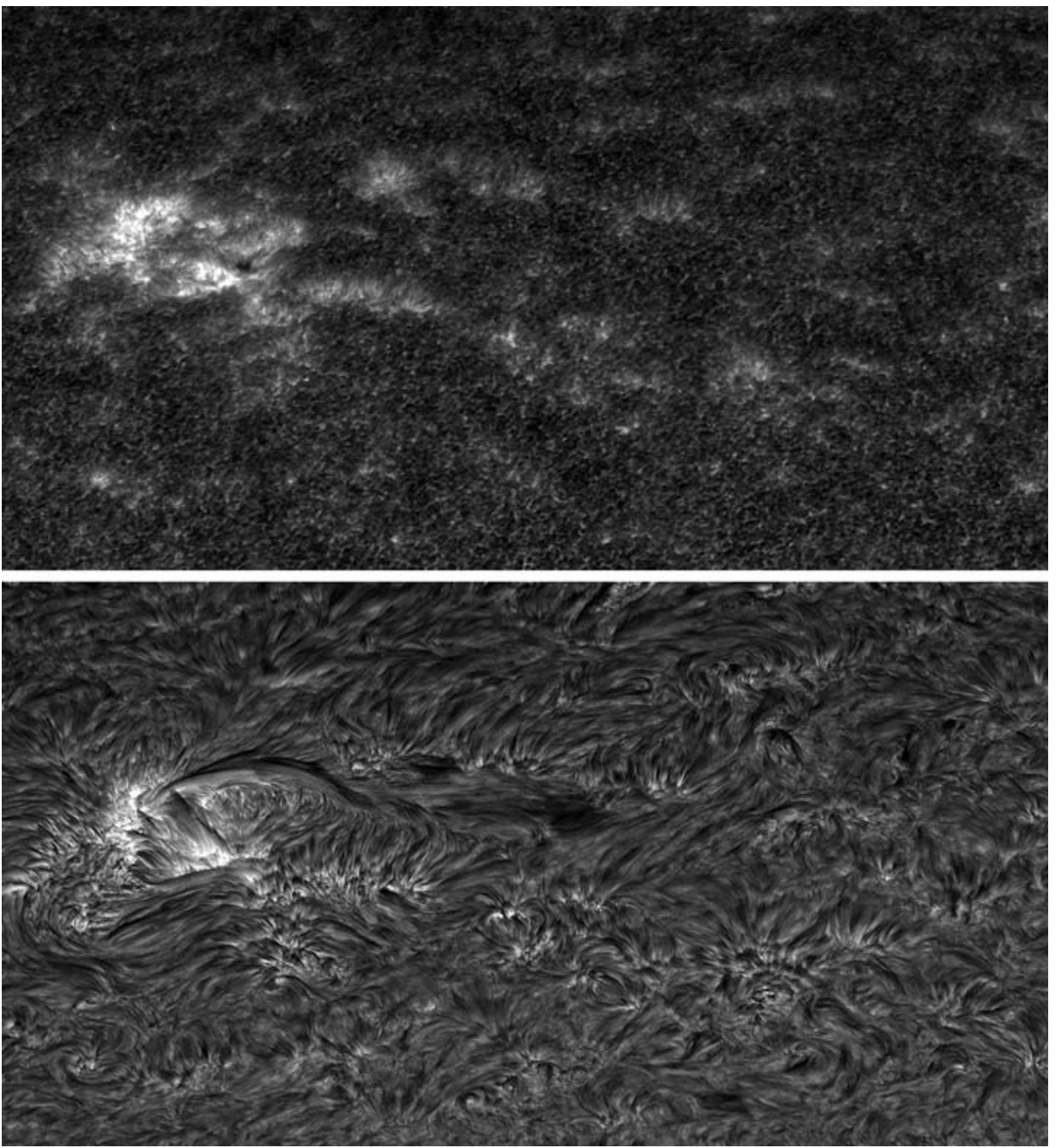

Figure 1 Simultaneous $\mathrm{Ca}$ II $\mathrm{H}$ and $\mathrm{H} \alpha$ image mosaics taken with the Dutch Open Telescope on 4 October 2005. The field of view is close to the limb (located off the top) and measures about $265 \times 143 \mathrm{arcsec}^{2}$. The spectral passbands are FWHM $1.4 \AA$ for Ca II H and $0.25 \AA$ for $\mathrm{H} \alpha$. The same area was observed with the SST and described by van Noort and Rouppe van der Voort (2006). High-resolution images of Figures 1-6 are available as Electronic Supplementary Material at http://dx.doi.org/10.1007/s11207-008-9116-9.

shorter, highly dynamic loops and grainy brightness patterns that are not connected to the network (see also Rouppe van der Voort et al., 2007). None of these structures is observed in the upper panel, except for the straws that correspond to bright $\mathrm{H} \alpha$ fibril endings.

What about the spectral passbands in this comparison? The Doppler cores of $\mathrm{H}$ and $\mathrm{K}$ are much narrower than for $\mathrm{H} \alpha$, but $\mathrm{H}$ and $\mathrm{K}$ filter bandpasses are usually wider with FWHMs of $3 \AA$ for Hinode, $1.4 \AA$ for the DOT, $0.6 \AA$ for the Lyot filter at the German VTT (e.g., Tritschler et al., 2007), $0.3 \AA$ for the Lockheed-Martin filter at the former SVST (Brandt et al., 1992; Lites, Rutten, and Berger, 1999), and also $0.3 \AA$ for the Halle filter at the NSO/SP/DST (e.g., Bloomfield et al., 2004, who assigned propagation speeds and 


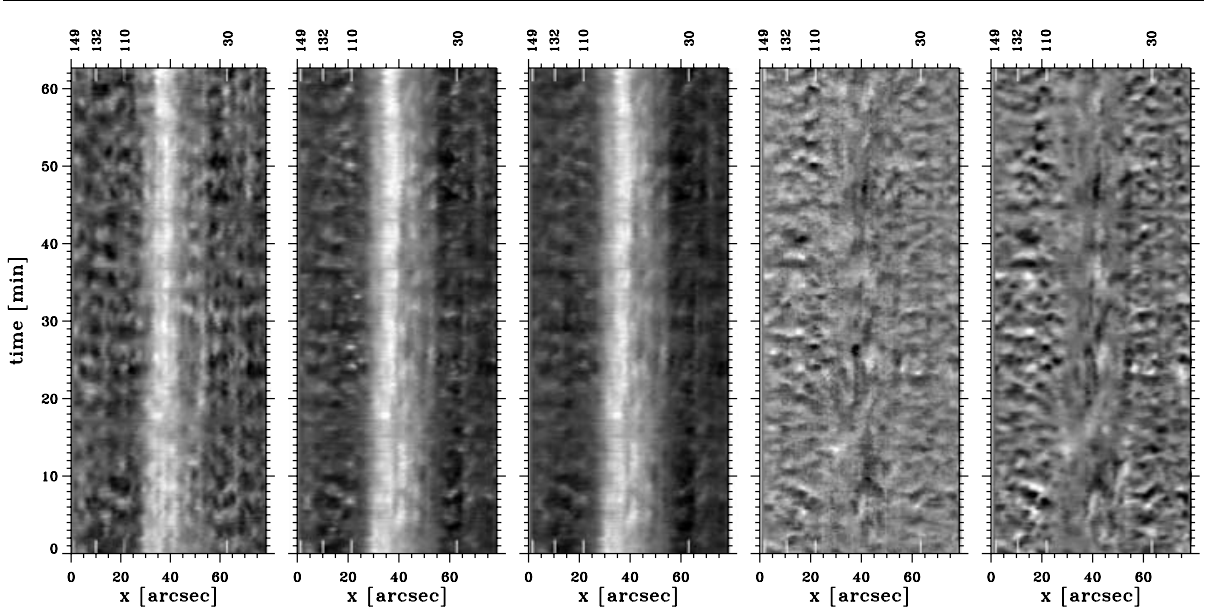

Figure 2 Various diagnostic measures of Ca II H plotted as space-time "time slice" evolution plots, assembled by B.W. Lites from a spectral sequence taken by him and W. Kalkofen in 1984 with the Dunn Solar Telescope at the National Solar Observatory/Sacramento Peak. The waves in the network part (bright strip at the center) were analyzed by Lites, Rutten, and Kalkofen (1993). The waves in the internetwork columns identified by numbers along the top were numerically simulated by Carlsson and Stein (1997). The spatial coordinate is measured along the slit of the spectrograph. The first three panels have logarithmic gray scales to reduce the large contrast between network and internetwork. Horizontal grayish "erasures" are due to poorer seeing. First panel: Intensity integrated over a spectral band of $0.9 \AA$ width centered on line center. Second panel: The same for $0.16 \AA$ bandpass. Third panel: Intensity of the core-profile minimum. Fourth panel: Wavelength variation of the core-profile minimum. Fifth panel: Dopplergram ratio $(R-V) /(R+V)$, with $R$ and $V$ being the intensities in $0.16 \AA$ passbands at $\mathrm{H}_{2 \mathrm{R}}$ and $\mathrm{H}_{2 \mathrm{~V}}$.

mode conversions to chromospheric oscillations by simply adopting the VAL3C line-center height difference). Such $\mathrm{H}$ and $\mathrm{K}$ filter imaging adds considerable inner-wing contributions from the upper photosphere, including bright reversed granulation and yet brighter acoustic grains, which may wash out the line-center-only contribution from chromospheric fibrils in the internetwork except where these are very bright, as in straws. The narrowest band images with high spatial resolution published so far are R.A. Shine's $0.3 \AA$ ones in Lites, Rutten, and Berger (1999), which only show reversed granulation and acoustic grains in the internetwork. Narrow-band $\mathrm{K}_{3}$ spectroheliograms such as the $0.1 \AA$ ones in the collection of Title (1966) have lower angular resolution but do not suggest the presence of similar masses of internetwork $\mathrm{Ca}$ II $\mathrm{K}$ fibrils as seen in $\mathrm{H} \alpha$ at similar resolution, except near active network and plage.

Figure 2 extends the Ca II H passband comparisons in Figure 1 of Rutten, De Pontieu, and Lites (1999) and Figure 9 of Krijger et al. (2001) with additional spectral measures from the ancient but high-quality Ca II H spectrogram sequence of Lites, Rutten, and Kalkofen (1993), which was also used by Carlsson and Stein (1997). The first three panels show that a narrower $\mathrm{Ca}$ II $\mathrm{H}$ passband produces larger network-to-internetwork brightness contrast but does not significantly change the morphology of the observed scene. The oscillation patterns in the internetwork, brightest at $\mathrm{H}_{2 \mathrm{~V}}$, remain visible even in the line-core intensity in the third panel. The line-core shift in the fourth panel confirms the acoustic nature of these patterns. Thus, even at full spectral resolution, the $\mathrm{Ca}$ II $\mathrm{H}$ core in this internetwork sample is dominated by radial three-minute waves, without obliteration by overlying fibrils or fibrilaligned motions. The final panel showing the $\mathrm{H}_{2 \mathrm{R}}-\mathrm{H}_{2 \mathrm{~V}}$ Dopplergram ratio strengthens this conclusion. This measure comes closest to exhibiting chromospheric-fibril structure in the 
form of oscillatory branches jutting out from the network with time, but regular three-minute oscillation patterns dominate further away in the internetwork.

The lower left panel of Figure 21 of Krijger et al. (2001) demonstrated the close correspondence between $\mathrm{Ca} I \mathrm{H}$ line-center dynamics and underlying photospheric dynamics in the internetwork parts of these spectrograms in Fourier terms. In fact, it would be strange if the line-center formation layer would not fully partake in the shock dynamics in these data because substantial in-phase upper atmosphere downdraft, above the next upcoming grain-causing acoustic shock, is required to give the $\mathrm{H}_{2 \mathrm{~V}}$ and $\mathrm{K}_{2 \mathrm{~V}}$ grains their characteristic spectral asymmetry, as shown in the formation breakdown diagrams of Carlsson and Stein (1997). They actually obtained their best sequence reproduction for near-network column 110 in Figure 2, suggesting that even there the field was too weak to upset $\mathrm{H}_{3}$ acoustic shock signatures.

Thus, these well-studied Ca II H spectrograms contradict the presence of opaque chromospheric fibrils masking upper photosphere (or "clapotispheric") internetwork dynamics in $\mathrm{H}$ and $\mathrm{K}$, whereas $\mathrm{H} \alpha$ canopy fibrils ubiquitously do so. The notion that the Ca II core generally forms higher than the $\mathrm{H} \alpha$ core, or that chromospheric structures should generally be more opaque in $\mathrm{H}$ and $\mathrm{K}$ than in $\mathrm{H} \alpha$, seems questionable. The observations suggest instead that the fibrils that constitute the $\mathrm{H} \alpha$ chromosphere are either transparent in $\mathrm{H}$ and $\mathrm{K}$ or, when opaque, are either pummeled by shocks from below without hindrance (such as magnetic tension) or represent a featureless, very dark blanket located well above the clapotisphere.

In summary, we suspect that much of the $\mathrm{K}_{3}$ internetwork dynamics is dominated by shock modulation at heights around $1000 \mathrm{~km}$, well below the VAL3C $\mathrm{K}_{3}$ formation height of $1800-2000 \mathrm{~km}$ and far below most $\mathrm{H} \alpha$ canopy fibrils.

This large discrepancy with equilibrium modeling may become understandable with the recent simulation of Leenaarts et al. (2007) implementing noninstantaneous hydrogen ionization and recombination. Three aspects combine in such an explanation. First, the simulated chromosphere is pervaded by shocks. Second, the large difference between hydrogen ionization/recombination balancing speed in hot shocks and in cool post-shock gas implies that instantaneous statistical equilibrium does not apply to the latter. Carlsson and Stein (2002) already explained why this is so. The large 10-eV excitation energy of its $n=2$ level causes hydrogen to be ionized rapidly in shocks but to recombine slowly in the cool intershock phases so that the ionization degree remains high also in the latter. Third, the population of H $\alpha$ 's lower $n=2$ level is closely coupled to the ion population (through the Balmer continuum; see also Rutten and Carlsson, 1994) and therefore remains similarly high at low post-shock temperature. Thus, the large $n=2$ excitation energy, being the very reason why $\mathrm{H} \alpha$ has very small cool-gas opacity in Boltzmann balancing, slows down the hydrogen recombination in post-shock cool gas so much that its $\mathrm{H} \alpha$ opacity exceeds the LTE value by many orders of magnitude. Therefore, fibrils can be opaque in $\mathrm{H} \alpha$ whether they are shock-hot or post-shock cool, and they even may be more opaque in $\mathrm{H} \alpha$ than in $\mathrm{H}$ and $\mathrm{K}$ at any temperature - in utter conflict with equilibrium modeling.

The chromospheric nonequilibrium balancing between Ca II (12-eV ionization energy) and Ca III is probably faster (with no top-heavy term diagram) but has not yet been analyzed in similar detail; it is of interest to evaluate the opacity ratio between $\mathrm{H}$ and $\mathrm{K}$ and $\mathrm{H} \alpha$ in dynamic conditions. Another point of interest is that chromospheric fibrils seem to be more obvious in Ca II $8542 \AA$ than in $\mathrm{H}_{3}$ and $\mathrm{K}_{3}$, possibly because of larger Doppler sensitivity (Cauzzi et al., 2007).

Thus, $\mathrm{H} \alpha$ is the key diagnostic of the internetwork chromosphere. Recent analyses of the chromosphere observed at high cadence in $\mathrm{H} \alpha$ line center with the Swedish 1-m Solar 
Telescope (SST) have shed new light on chromospheric fibrils, showing that near network and plage "dynamic fibrils" tend to have repetitive mass loading at three- to five-minute shock periodicity (De Pontieu et al., 2007) whereas the little loops in quiet-Sun internetwork are even more dynamic, presumably because of stronger buffeting by shocks where the magnetic field is weak (Rouppe van der Voort et al., 2007).

In this paper we use image sequences from the DOT with lower cadence than $\mathrm{H} \alpha$ really requires (van Noort and Rouppe van der Voort, 2006), but with $\mathrm{H} \alpha$ profile sampling and synchronous co-spatial imaging in other wavelengths including $\mathrm{Ca}$ II $\mathrm{H}$. These images permit us to isolate specific events in the photosphere and inspect the chromospheric response in the apparent brightness of $\mathrm{Ca}$ II $\mathrm{H}$ and $\mathrm{H} \alpha$, and particularly in $\mathrm{H} \alpha$ Doppler modulation. We do this here by presenting three exemplary cases: an acoustic grain, a persistent flasher, and an exploding granule.

\section{Observations and Reduction}

The image sequences used here were obtained with the DOT on La Palma on 14 October 2005 during 10:15:43 - 10:30:42 UT. The telescope, instrumentation, and data processing are described by Rutten et al. (2004).

Synchronous and co-spatial images were taken in blue and red continuum passbands, in the $\mathrm{G}$ band around $4305 \AA$, in Ca II H alternatingly at line center and at $\Delta \lambda=-1 \AA$, and at five $\mathrm{H} \alpha$ wavelengths: line center, the $\mathrm{H} \alpha \pm 350 \mathrm{~m} \AA$ inner wing pair, and the $H \alpha \pm 700 \mathrm{~m} \AA$ outer wing pair. The seeing had mean Fried parameter $r_{0}=7 \mathrm{~cm}$ for the $\mathrm{G}$ band, just about good enough that the standard DOT speckle reconstruction produced angular resolution close to the diffraction limit of the $45-\mathrm{cm}$ aperture ( 0.2 arcsec in the blue). The field of view measured $79 \times 60 \operatorname{arcsec}^{2}\left(56 \times 43 \mathrm{Mm}^{2}\right)$, covering a very quiet area at disk center containing only few and sparse weak-network clusters of magnetic elements (bright points in the $\mathrm{G}$ band, bright grain clusters in $\mathrm{Ca}$ II $\mathrm{H}$ ), as demonstrated by the first two sample images in Figure 3. This area was sufficiently quiet that $\mathrm{H} \alpha$ shows only short, highly dynamic fibrils concentrated around the magnetic clusters.

The image sequences had slightly irregular cadence and were interpolated to 30-image sequences at strict 30-second cadence. The G-band images were subsonically Fourierfiltered in $(k, \omega)$ space, passing only components with apparent motion below the sound speed of $7 \mathrm{~km} \mathrm{~s}^{-1}$ to remove the brightness modulation owing to the $p$-mode oscillations since in this study granular dynamics is emphasized as a pistoning agent. Horizontal flows were measured by using the cross-correlation tracking algorithm of November (1986) and November and Simon (1988), with Gaussian FWHM widths of 1.5 arcsec and 330 seconds as boxcar averaging parameters. Differences between neighboring pixels were used to evaluate derivatives to determine the local horizontal divergence and vorticity in the flows.

The $\mathrm{H} \alpha$ sequences were also cone-filtered in $(k, \omega)$ space but in this case reversely, only passing the supersonic components because here the oscillatory response of the chromosphere is of interest. Our intention was to remove stable fibrils to enhance the visibility of oscillatory modulations such as spreading rings, but in this very quiet area the fibrils are so dynamic that the differences between the filtered and unfiltered sequences are small. Finally, $\mathrm{H} \alpha$ Dopplergrams were constructed from the filtered $\mathrm{H} \alpha \pm 700 \mathrm{~m} \AA$ and $\mathrm{H} \alpha \pm 350 \mathrm{~m} \AA$ image pairs.

We used a live, multipanel, on-screen display similar to Figures 4-6 to inspect the various diagnostics simultaneously and in mutual correspondence at large local magnification, varying both the location and time coordinates of the point of scrutiny within these image 

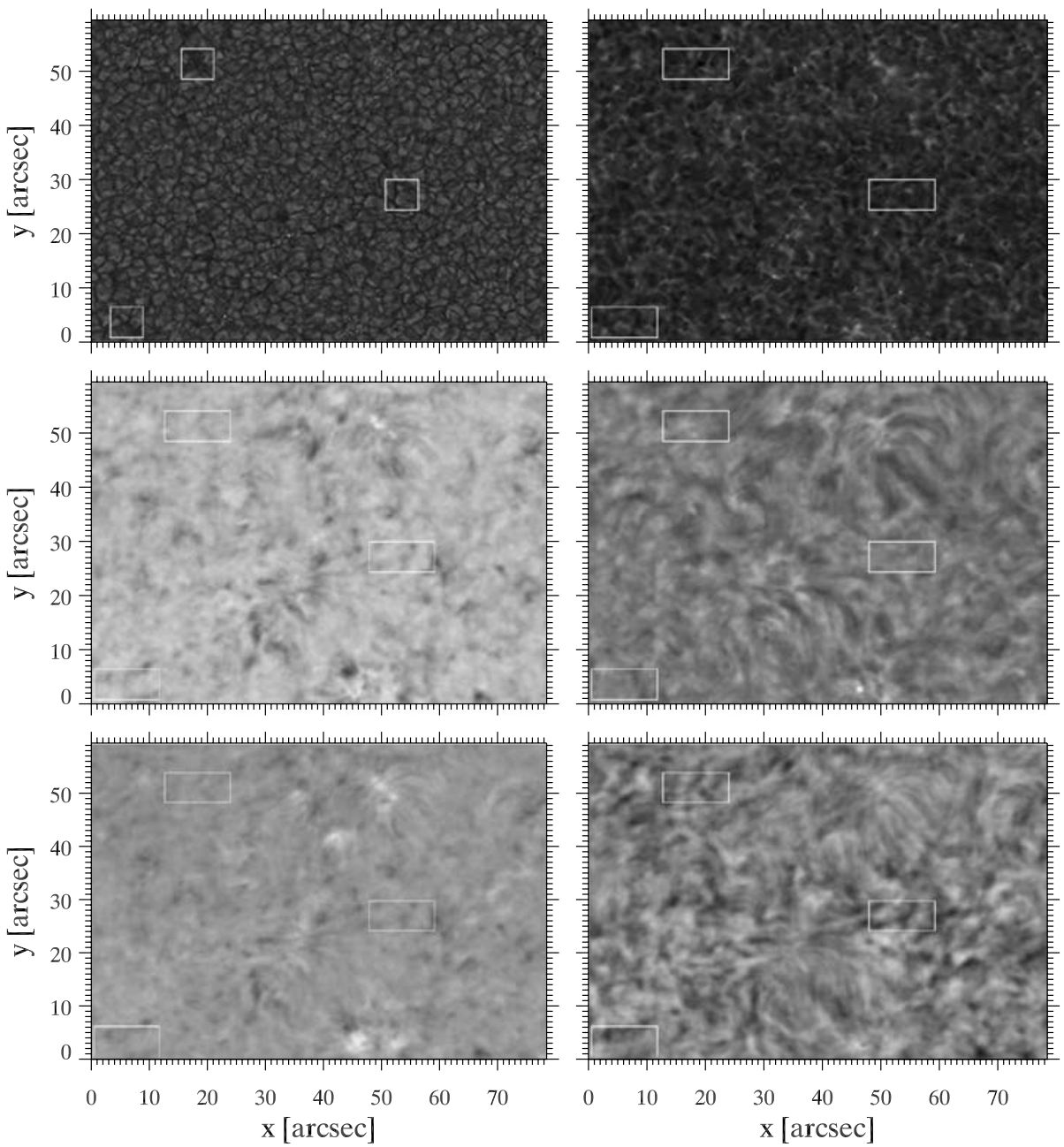

Figure 3 Simultaneous sample images (from top left across to bottom right): $\mathrm{G}$ band, Ca II $\mathrm{H}$ line center, $\mathrm{H} \alpha-700 \mathrm{m \AA}, \mathrm{H} \alpha$ line center, and $\mathrm{H} \alpha \pm 700 \mathrm{~m} \AA$ and $\mathrm{H} \alpha \pm 350 \mathrm{~m} \AA$ Dopplergrams with bright areas implying downdraft. The G-band image is Fourier-filtered to pass only subsonic components. The $\mathrm{H} \alpha$ images and Dopplergrams are filtered to pass only supersonic components. The white boxes specify the three subfields analyzed in Figures 4-6, from left to right respectively. The cutout boxes are larger for Ca II $\mathrm{H}$ and $\mathrm{H} \alpha$ to show more spatial context in the time slices of Figures $4-6$.

sequences. The three cases discussed in the following have been selected in this subjective manner as being the most informative - they are not "typical" but are the "best" examples. However, because the field of view and the sequence duration were small, they are nevertheless indicative of common occurrences on the solar surface. Automation of such a vision-based selection process for larger data sets is nontrivial.

Figure 3 shows that all three examples lie in pure internetwork without magnetic elements and with grainy rather than fibrilar $\mathrm{H} \alpha$ fine structure. They sample the thinnest and lowest sort of $\mathrm{H} \alpha$ chromosphere. 

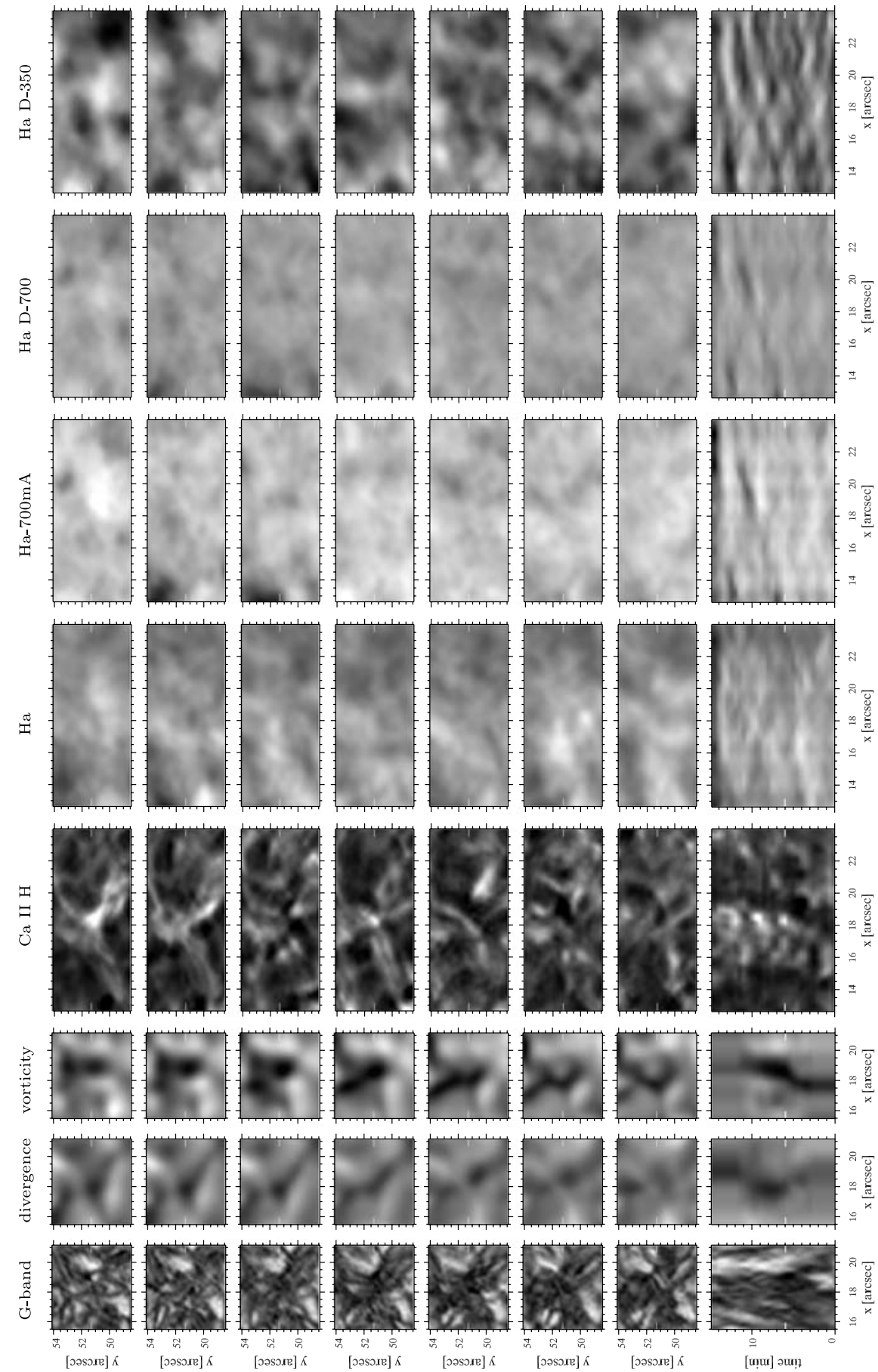
Figure 4 Acoustic $\mathrm{H}_{2 V}$ grain. The first seven rows are small image cutouts, in this case corresponding to the leftmost box in Figure 3. Each panel is spatially centered on the acoustic grain. The diagnostics are specified at the top; the last two columns are the $\mathrm{H} \alpha \pm 700 \mathrm{~m} \AA$ and $\mathrm{H} \alpha \pm 350 \mathrm{~m} \AA$ Dopplergrams. The cutouts are wider for the upper atmosphere diagnostics ( $\mathrm{Ca}$ II $\mathrm{H}$ and $\mathrm{H} \alpha$ ) to show more context in their $x-t$ time slices at the bottom. The latter show the brightness evolution along the horizontal cut through the center of the subfield defined by the white markers in each image cutout. The time step between the consecutive image rows is one minute, with time increasing upward to correspond with the time direction of the slices. The image cutout sequences span seven minutes centered on the first appearance of the grain (bright small Ca II $\mathrm{H}$ blob in the fourth column and fourth row). In the second column bright and dark measure divergence and convergence, respectively. In the third column bright implies counterclockwise rotation. In the Dopplergrams (last two columns) bright implies downdraft. The gray scaling is the same per column throughout Figures 4-6 to enable intercomparison. The time slices have been temporally interpolated to 15 -second cadence to gain display height.

\section{Acoustic Grains}

Figure 4 shows the appearance of a regular $\mathrm{Ca} I \mathrm{I} \mathrm{H}_{2 \mathrm{~V}}$ "grain train." The caption specifies the complex figure layout (which also applies for Figures 5 and 6). The cutout image sequences (with time increasing upward in compliance with the $x-t$ time slices at the bottom) are centered on the sudden appearance of a $\mathrm{Ca}$ II $\mathrm{H}$ grain (fourth row, fourth column). It reappeared three and six minutes later, marking a regular $\mathrm{H}_{2 \mathrm{~V}}$ shock sequence much like the triple grain occurrence at the top of column 30 in the first panel of Figure 2, shown also in Figures 9 and 10 of Krijger et al. (2001) and Fourier-analyzed there in detail, where it was noted that such clear three-minute periodicity requires the absence of large five-minute modulation but spatial coincidence with the slower evolving reversed-granulation background pattern. The latter was subsequently analyzed by Rutten, De Wijn, and Sütterlin (2004), Leenaarts and Wedemeyer-Böhm (2005), and Cheung, Schüssler, and Moreno-Insertis (2007). Such spatial coincidence is indeed seen to be the case in the Ca II H cutouts and time slice in Figure 4. (Note that reversed granulation is not seen in $\mathrm{H} \alpha$, which has insufficient opacity in the middle photosphere.) These three grains undoubtedly mark acoustic shocks such as the ones simulated by Carlsson and Stein (1997), including the column-30 ones of Figure 2 (their Figure 17, Plate 21).

Do these Ca II $\mathrm{H}$ grains mark an acoustic event? If so, one expects that a small granular feature disappeared about two minutes before the grain train onset. The corresponding area does show an intergranular space in the wake of a bright granule that split apart and disappeared two-five minutes earlier (seen in the G-band time slice, not fully covered by the cutout sequence). Before the grain appeared there was weak divergence meeting weak convergence at the splitting-granule site, eventually followed by stronger convergence that bordered marked vorticity (dark blobs in the upper panels of the second and third columns), which earlier was located in the lane besides the splitting granule (third time slice). Together, the photospheric diagnostics suggest that these grains mark acoustic excitation by a small whirlpool downdraft.

What happened in the overlying chromosphere? This subfield is located, as are the other two, in quiet internetwork without clear $\mathrm{H} \alpha$ fibrils. The $\mathrm{H} \alpha$ line-center column shows nothing special at the grain location. The top $\mathrm{H} \alpha-700 \mathrm{~m} \AA$ panel shows marked brightening there, followed within two minutes by yet stronger darkening (seen in the time slice, which extends longer). The same pattern appears in the $\mathrm{H} \alpha \pm 700 \mathrm{~m} \AA$ Doppler time slice, so this dark-after-bright pattern is mostly Doppler modulation, shifting the line out and into the passband. Because of the curvature of the line profile such Doppler darkening tends to be stronger than counterphase brightening, as is the case here. The same bright-dark pair is also recognizable in the $\mathrm{H} \alpha \pm 350 \mathrm{~m} \AA$ time slice, more clearly preceded by another, smaller 
bright - dark pair at the grain location, and with apparent connectivity to longer features toward the top of the time slice (beyond the top cutout images), extending over four to five arcseconds. Comparison with the $\mathrm{H} \alpha \pm 700 \mathrm{~m} \AA$ time slice shows that these are weakly present there as well. Their extent might suggest wide wave spreading with height, but the Dopplergram cutouts do not show corresponding rings around the grain location. Either the spreading wave ran into a finely structured atmosphere or made already-existing $\mathrm{H} \alpha$ fibrils shake over extended lengths through shock buffeting. If so, these fibrils are not very distinct in $\mathrm{H} \alpha$ line center (nor in the unfiltered images) and obtain their clearest mark of presence through Doppler modulation.

Note that the simultaneous appearance of the second $\mathrm{Ca}$ II $\mathrm{H}$ grain and the marked $\mathrm{H} \alpha-700 \mathrm{~m} \AA$ downdraft in the top row fits the scenario for spectrally asymmetric grain formation of Carlsson and Stein (1997). Comparison of the Ca II $\mathrm{H}$ and $\mathrm{H \alpha} \pm 350 \mathrm{~m} \AA$ time slices confirms that all three Ca II $\mathrm{H}$ grains indeed had concurrent chromospheric downdrafts.

\section{Persistent Flasher}

Figure 5 is again centered on the first appearance of a bright repetitive $\mathrm{Ca}$ II $\mathrm{H}$ grain, but this one had continuous brightness underlying its repeated brightening and was accompanied by a small bright point in the G-band images and time slice. This behavior suggests that it was a "persistent flasher," marking the presence of a small internetwork magnetic flux concentration (Brandt et al. 1992, 1994). These are more easily located in Ca II H image sequences than as G-band bright points and tend to come and go in the form of intermittent "magnetic patches," appearing as bright grains when squeezed together and vanishing when their concentration becomes less dense as imposed by the granular flows advecting their field (De Wijn et al., 2005). Neither the Ca II H flasher nor the G-band bright point is seen in the bottom half of the time slices, indicating that the concentration was initially more spread out. The second time slice indeed shows persistent convergence, which is already evident from the squeezing together of the large granules in the first time slice. The largest convergence is in the neighboring lanes, up to the bright-point appearance (lower cutouts in the second column). Another speculation might be the incidence of flux-tube collapse, but the convergence should then maximize at the bright-point site.

The chromosphere shows marked response in the $\mathrm{H} \alpha-700 \mathrm{~m} \AA$ and Doppler columns: a dark blob in the second row, which again is seen to result from an up - down dark - bright Doppler stroke in the time slices, most clearly in the $\mathrm{H} \alpha \pm 350 \mathrm{~m} \AA$ Doppler slice in the last column (partially mimicked at lower contrast by the $\mathrm{H} \alpha$ line-center intensity in the fifth slice), but in this case preceded by a brief bright downstroke. The size of the oscillatory blob is much larger than the Ca II $\mathrm{H}$ grains, especially in the second row, again suggesting response by extended fibrilar structures. The second row also indicates strong updraft above the (very sharp) Ca II $\mathrm{H}$ grain, suggesting a mechanism other than acoustic $\mathrm{H}_{2 \mathrm{~V}}$ grain formation. Fibril loading from the granular squeezing seen in the first time slice, sending gas upward along the field concentration indicated by the Ca II H flasher, may occur here following the scenario suggested by Babcock and Babcock (1955).

\section{Exploding Granule}

Our third example in Figure 6 concerns an exploding granule, to assess the suggestion of Rast (1995) that such features may act as acoustic sources. The cutout sequences are centered on the moment at which the marked divergence in the second time slice ends. The 


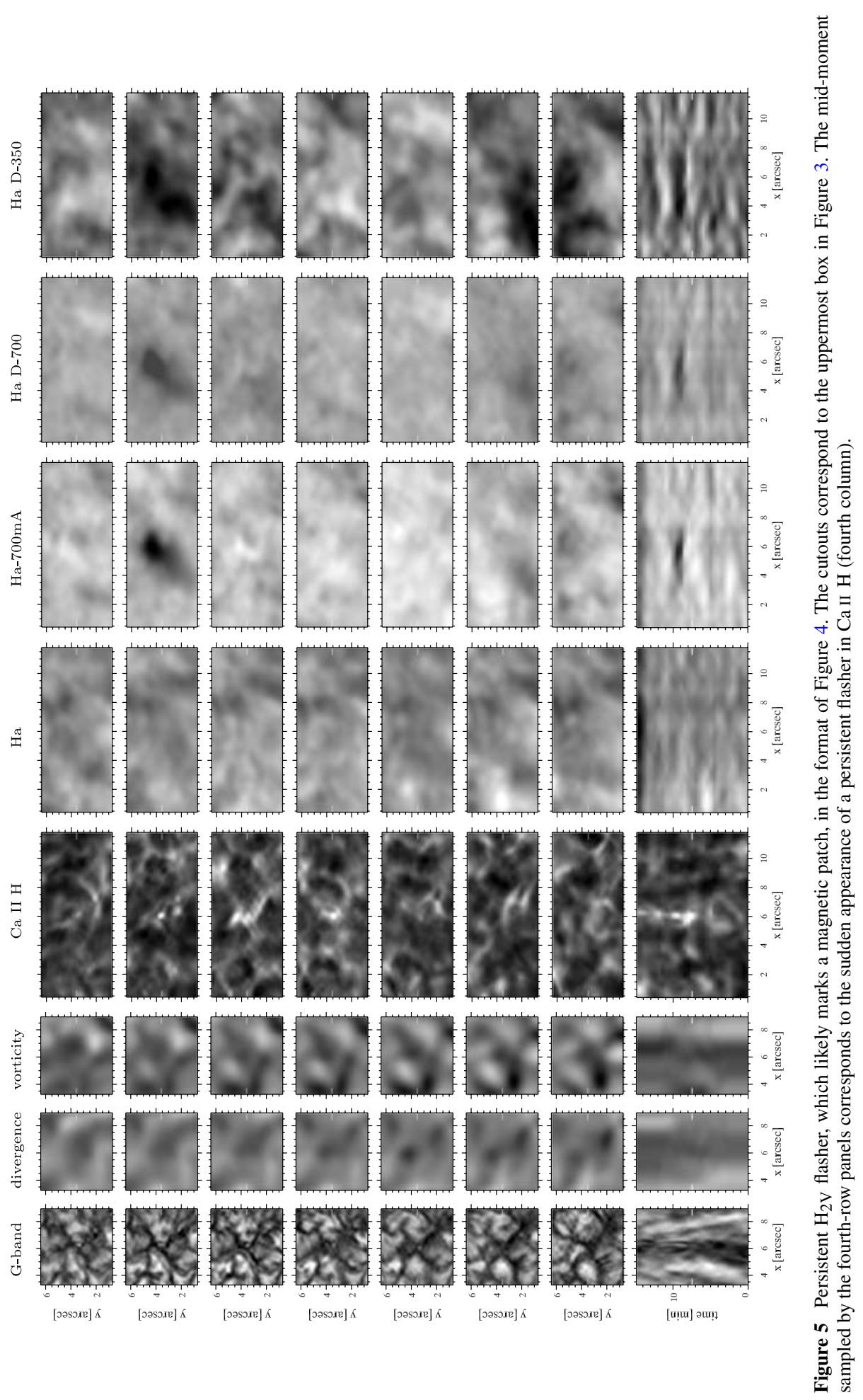



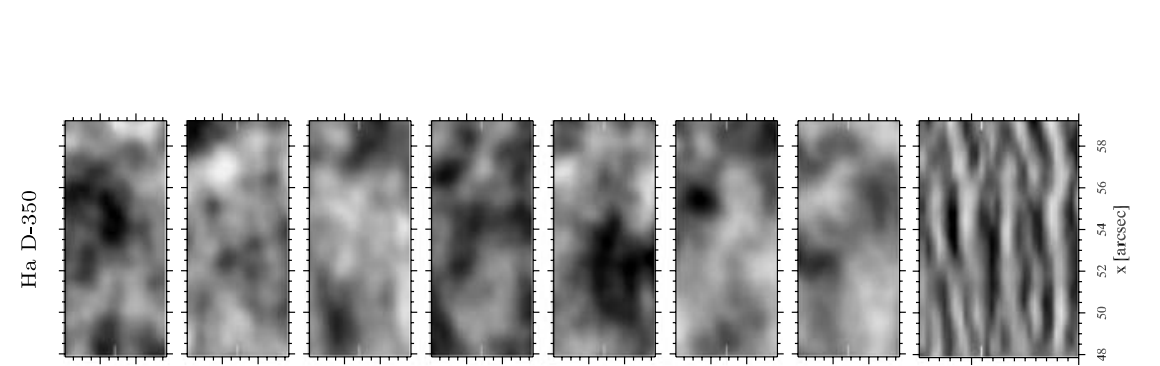

$\stackrel{Ð}{\Xi}$
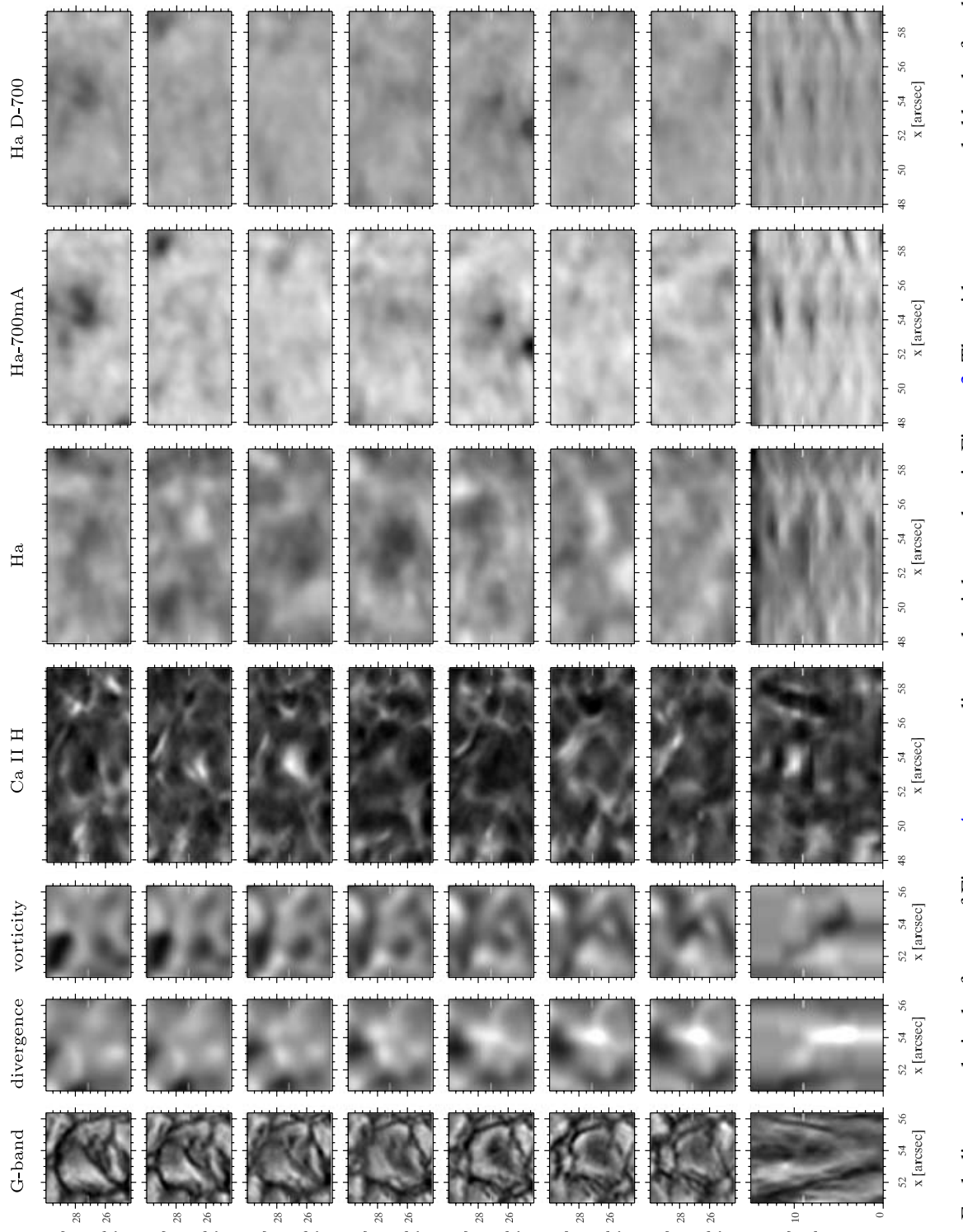

[วәรวงе] $K$

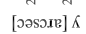

का

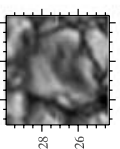

is
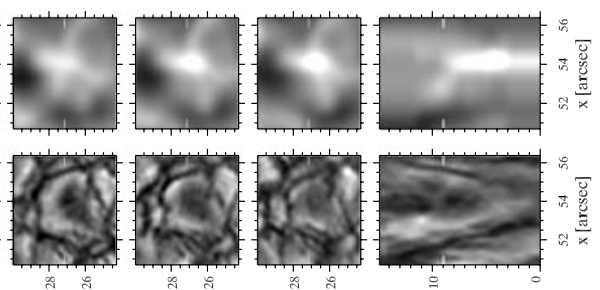

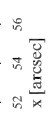
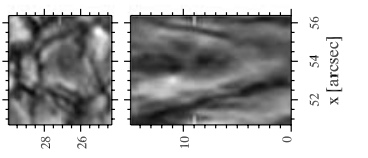

[ụ!] әu!̣

缡

6 हี

논 
granule had already a large dark center and was about to become three small granules with some shards in between. A minute later, the $\mathrm{Ca}$ II $\mathrm{H}$ line became very bright at that location, and after two more minutes very dark (top row). The same dark blob is seen along the top row in the $\mathrm{H} \alpha$ wing columns, implying large updraft. The $\mathrm{H} \alpha \pm 350 \mathrm{~m} \AA$ Doppler time slice in the last panel again shows much more spatially extended oscillatory behavior than the other diagnostics, suggesting fibril buffeting. The $\mathrm{H} \alpha$ line-center intensity time slice again mimics the Doppler behavior partially. The exploding granule seems to cause an oscillation amplitude increase above it that modulates the $\mathrm{H} \alpha$ core intensity.

\section{Discussion}

Each example shows a large morphological difference among the low-photosphere scene in the $\mathrm{G}$ band, the high-photosphere scene in $\mathrm{Ca}$ II $\mathrm{H}$, and the chromospheric scene in $\mathrm{H} \alpha$. The $\mathrm{H} \alpha$ cutouts do not contain internetwork-spanning fibrils in this quiet region; each of the three events therefore shows chromospheric response that would otherwise have been blocked by overlying canopy fibrils. The responses are not very striking; in no case may one locate the photospheric happening uniquely from its subsequent $\mathrm{H} \alpha$ signature.

The $\mathrm{H} \alpha$ chromosphere sampled by the image cutouts comes close to our tentative description of the $\mathrm{Ca} I \mathrm{H} \mathrm{H}_{3}$ and $\mathrm{K}_{3}$ internetwork chromosphere as a featureless opaque blanket pummeled from below. They may indeed be about the same, as suggested by the H $\alpha$ downdrafts in Figure 4 above grains requiring $\mathrm{H}_{3}$ downdraft for $\mathrm{H}_{2 \mathrm{~V}}$ profile asymmetry. The much smaller temperature sensitivity of the $\mathrm{H}$ and $\mathrm{K}$ opacity then indeed diminishes the $\mathrm{H}$ and $\mathrm{K}$ feature contrast. Because Ca II 8542 is an excited and narrower line, it combines larger temperature and Doppler sensitivity to such pummeling but at smaller blanket opacity.

Fibrilar structuring appears most clearly in the $\mathrm{H} \alpha$ Dopplergram time slices. Our impression is that indeed this quiet $\mathrm{H} \alpha$ chromosphere is continuously pummeled by internetwork waves, gaining much dynamics from these in the quietest areas where the magnetic fields have low tension but yet enough to impose slight fibrilarity. We suspect that slanted internalgravity waves contribute significantly in this pummeling (see Lighthill, 1967, pp. 440-443) and, together with the primarily vertically growing acoustic shocks, cause intricate and fastchanging interference patterns at granular to mesogranular scales. Only the fiercest events punch through to be individually recognizable directly above their sources.

Our three examples whet the appetite for even more comprehensive multidiagnostic solar-atmosphere tomography. The complexity of the panel layout in Figures $4-6$ already demonstrates that the dynamical coupling between photosphere and chromosphere can only be addressed holistically with diverse diagnostics. The complexity of the solar scenes shown in these panels and their large differences among diagnostics strengthen this conclusion. In fact, our data are yet incomplete: we lack the principal acoustic-event measure of "acoustic flux" in this analysis, requiring Fabry-Perot Doppler mapping of the photosphere at multiple heights. Sensitive photospheric magnetic field mapping and ultraviolet chromospheric image and Doppler diagnostics would also be welcome - preferably all at similar or better angular resolution, cadence, duration, and field size than used here.

In this paper we have limited the discussion to three, hand-picked cases from an early set of tomographic DOT image sequences. The large DOT database collected in the meantime (openly available at http://dotdb.phys.uu.nl/DOT) now permits wider statistical study of such various types of events. Rapid-cadence Fabry - Perot imaging at the SST promises unprecedented $\mathrm{H} \alpha$ diagnostics. Space-mission co-pointing adds EUV diagnostics of the transition region. Pertinent numerical simulations including realistic formation of $\mathrm{H} \alpha$ are also coming into reach. 
Acknowledgements The DOT is owned by Utrecht University and located at the Spanish Observatorio del Roque de los Muchachos of the Instituto de Astrofísica de Canarias. We are deeply indebted to V. Gaizauskas for the DOT H $\alpha$ filter. We thank M. Carlsson, Ø. Langangen, S.E.M. Keek, J.M.D. Kruijssen, and A.G. de Wijn for inspiring debates. This research made much use of SST hospitality and of NASA's Astrophysics Data System. R.J. Rutten thanks the Leids Kerkhoven-Bosscha Fonds and the organizers of the SOHO 19/GONG 2007 meeting for travel support.

Open Access This article is distributed under the terms of the Creative Commons Attribution Noncommercial License which permits any noncommercial use, distribution, and reproduction in any medium, provided the original author(s) and source are credited.

\section{References}

Babcock, H.W., Babcock, H.D.: 1955, Astrophys. J. 121, 349.

Bloomfield, D.S., McAteer, R.T.J., Mathioudakis, M., Williams, D.R., Keenan, F.P.: 2004, Astrophys. J. 604, 936.

Brandt, P.N., Rutten, R.J., Shine, R.A., Trujillo Bueno, J.: 1992, In: Giampapa, M.S., Bookbinder, J.A. (eds.) Cool Stars, Stellar Systems, and the Sun, Proc. Seventh Cambridge Workshop 26, ASP, San Francisco, 161.

Brandt, P.N., Rutten, R.J., Shine, R.A., Trujillo Bueno, J.: 1994, In: Rutten, R.J., Schrijver, C.J. (eds.) Solar Surface Magnetism, NATO ASI Series C 433, Kluwer, Dordrecht, 251.

Carlsson, M., Stein, R.F.: 1994, In: Carlsson, M. (ed.) Chromospheric Dynamics. Proc. Miniworkshop, Inst. Theor. Astrophys., Oslo, 47.

Carlsson, M., Stein, R.F.: 1997, Astrophys. J. 481, 500.

Carlsson, M., Stein, R.F.: 2002, Astrophys. J. 572, 626.

Cauzzi G., Reardon K.P., Uitenbroek H., Cavallini F., Falchi A., Falciani R., Janssen K., Rimmele T., Vecchio A., Woeger F.: 2007, Astron. Astrophys. submitted. http://arxiv.org/abs/0709.2417.

Cheung, M.C.M., Schüssler, M., Moreno-Insertis, F.: 2007, Astron. Astrophys. 461, 1163.

De Pontieu, B., Hansteen, V.H., Rouppe van der Voort, L., van Noort, M., Carlsson, M.: 2007, Astrophys. J. 655, 624.

De Wijn, A.G., Rutten, R.J., Haverkamp, E.M.W.P., Sütterlin, P.: 2005, Astron. Astrophys. 441, 1183.

Goode, P.: 1995, STI/Recon Technical Report N 96, NASA, Washington, 10005.

Goode P.R.: 2002, Bull. Am. Astron. Soc. 34, 730.

Goode, P.R., Gough, D., Kosovichev, A.G.: 1992, Astrophys. J. 387, 707.

Goode, P.R., Strous, L.H., Rimmele, T.R., Stebbins, R.T.: 1998, Astrophys. J. 495, L27.

Hoekzema, N.M., Rimmele, T.R., Rutten, R.J.: 2002, Astron. Astrophys. 390, 681.

Krijger, J.M., Rutten, R.J., Lites, B.W., Straus, T., Shine, R.A., Tarbell, T.D.: 2001, Astron. Astrophys. 379, 1052.

Leenaarts, J., Wedemeyer-Böhm, S.: 2005, Astron. Astrophys. 431, 687.

Leenaarts, J., Rutten, R.J., Sütterlin, P., Carlsson, M., Uitenbroek, H.: 2006, Astron. Astrophys. 449, 1209.

Leenaarts, J., Carlsson, M., Hansteen, V., Rutten, R.J.: 2007, Astron. Astrophys. 473, 625.

Lighthill, M.J.: 1967, In: Thomas, R.N. (ed.) Aerodynamical Phenomena in Stellar Atmospheres, IAU Symp. 28, Academic Press, New York, 429.

Lites, B.W., Rutten, R.J., Kalkofen, W.: 1993, Astrophys. J. 414, 345.

Lites, B.W., Rutten, R.J., Berger, T.E.: 1999, Astrophys. J. 517, 1013.

November, L.J.: 1986, Appl. Opt. 25, 392.

November, L.J., Simon, G.W.: 1988, Astrophys. J. 333, 427.

Rast, M.P.: 1995, Astrophys. J. 443, 863.

Rast, M.P.: 1999, Astrophys. J. 524, 462.

Restaino, S.R., Stebbins, R.T., Goode, P.R.: 1993, Astrophys. J. 408, L57.

Rimmele, T.R., Goode, P.R., Harold, E., Stebbins, R.T.: 1995, Astrophys. J. 444, L119.

Rouppe van der Voort, L.H.M., De Pontieu, B., Hansteen, V.H., Carlsson, M., van Noort, M.: 2007, Astrophys. J. 660, L169.

Rutten R.J.: 2007, In: Heinzel, P., Dorotovič, I., Rutten, R.J. (eds.) The Physics of Chromospheric Plasmas, Conf. Series 368, ASP, San Francisco, 27.

Rutten R.J., Carlsson M.: 1994, In: Rabin, D.M., Jefferies, J.T., Lindsey, C (eds.) Infrared Solar Physics, IAU Symposium 154, Kluwer, Dordrecht, 309.

Rutten, R.J., Uitenbroek, H.: 1991, Solar Phys. 134, 15. 
Rutten R.J., De Pontieu B., Lites B.: 1999, In: Rimmele, T.R., Balasubramaniam, K.S., Radick, R.R. (eds.) High Resolution Solar Physics: Theory, Observations, and Techniques, Conf. Series 183, ASP, San Francisco, 383.

Rutten, R.J., De Wijn, A.G., Sütterlin, P.: 2004, Astron. Astrophys. 416, 333.

Rutten, R.J., Hammerschlag, R.H., Bettonvil, F.C.M., Sütterlin, P., De Wijn, A.G.: 2004, Astron. Astrophys. 413, 1183.

Skartlien, R., Rast, M.P.: 2000, Astrophys. J. 535, 464.

Skartlien, R., Stein, R.F., Nordlund, Å.: 2000, Astrophys. J. 541, 468.

Stebbins, R., Goode, P.R.: 1987, Solar Phys. 110, 237.

Strous, L.H., Goode, P.R., Rimmele, T.R.: 2000, Astrophys. J. 535, 1000.

Title A.M.: 1966, Selected Spectroheliograms, Mount Wilson and Palomar Observatories, Pasadena.

Tritschler, A., Schmidt, W., Uitenbroek, H., Wedemeyer-Böhm, S.: 2007, Astron. Astrophys. 462, 303.

van Noort, M.J., Rouppe van der Voort, L.H.M.: 2006, Astrophys. J. 648, L67.

Vernazza, J.E., Avrett, E.H., Loeser, R.: 1973, Astrophys. J. 184, 605.

Vernazza, J.E., Avrett, E.H., Loeser, R.: 1976, Astrophys. J. Suppl. Ser. 30, 1.

Vernazza, J.E., Avrett, E.H., Loeser, R.: 1981, Astrophys. J. Suppl. Ser. 45, 635. 